\title{
A Numerical Approach for Two Parallel Surface Cracks of infinite plane
}

\author{
Zhao Xiaohua, a and Liu Baoliang ${ }^{\text {2,b }}$ \\ ${ }^{1}$ China Building Technique Group Co., Ltd., Beijing 100013, P.R.China \\ ${ }^{2}$ Science college, Heilongjiang Institute of Science and Technology, Harbin 150022, P.R.China \\ azxh12_19@sina.com, baoliangliu2008@163.com
}

Keywords: Stress intensity factor, Displacement discontinuity, Parallel Cracks

Abstract. This paper deals with two Parallel Surface Cracks of infinite plane. By using a hybrid displacement discontinuity method (a boundary element method) proposed recently, two Parallel Surface Cracks of infinite plane models are analyzed in detail. By changing the geometrical forms and parameters of crack, which is a model frequently used in fracture mechanics, the effect of the geometrical forms and parameters of crack on the stress intensity factors (SIFs) of two Parallel Surface Cracks of infinite plane specimen, is revealed. Some geometric parameters are introduced here, which are used to formulate two Parallel Surface Cracks of infinite plane.

\section{Introduction}

Due to the stress concentration effect around the hole, cracks are likely to initiate at the hole under the action of fatigue loading. Consequently, a number of papers dealing with hole edge crack problems (called hole crack problems for short) are available, see Ref.[1].The hybrid displacement discontinuity method differs from hybrid boundary element, when used to analyze the SIFs of a branched crack, require the plate to be modeled as a finite plate of huge dimensions by fictitious stress elements, while the crack could be modeled by displacement discontinuity elements. This brings about a higher computational effort. While the hybrid displacement discontinuity method was used to analyze crack problem with very high accuracy and efficiency [2-3].

Pan [4] pointed out that the displacement discontinuity method is quite suitable for cracks in infinite domain where there is no no-crack boundary. However, it alone may not be efficient for finite domain problems, since the kernel functions in DDM involve singularities with order higher than those in the traditional displacement BEM". Recently, the hybrid displacement discontinuity method was used by Yan [5-6] to calculate the SIFs of complex plane cracks in a finite plate. The numerical results showed that the numerical approach was also simple, yet very accurate.

In addition, it was found from previous investigations that the hybrid displacement discontinuity method was both accurate and efficient for analyzing a multiple void-crack interaction [7-12].

\section{Brief Description of the Hybrid Displacement Discontinuity Method}

In this section, the hybrid displacement discontinuity method presented by Yan [13] is described briefly. It consists of the constant displacement discontinuity element presented by Crouch and Starfield [14] and the crack-tip displacement discontinuity elements.

\section{Constant Displacement Discontinuity Method}

The displacement discontinuity Di is defined as the difference in displacement between the two sides of the segment [14] (see Fig.1): $\boldsymbol{D}_{\boldsymbol{i}}=\boldsymbol{D}_{\boldsymbol{i}}(\xi) \quad(\boldsymbol{i}=1,2)$ 


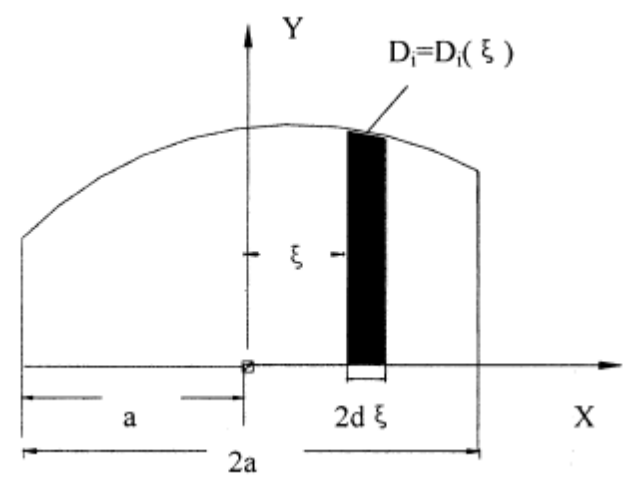

Fig.1 Schematic of constant displacement discontinuity components $D_{i}$

$$
\begin{aligned}
d u_{x} & =D_{x}(\xi)\left[2(1-v) T_{3}(x, y, \xi, d \xi)-y T_{5}(x, y, \xi, d \xi)\right] \\
& +D_{y}(\xi)\left[-(1-2 v) T_{2}(x, y, \xi, d \xi)-y T_{4}(x, y, \xi, d \xi)\right] \\
d u_{y} & =D_{x}(\xi)\left[(1-2 v) T_{2}(x, y, \xi, d \xi)-y T_{4}(x, y, \xi, d \xi)\right] \\
& +D_{y}(\xi)\left[2(1-v) T_{3}(x, y, \xi, d \xi)-y T_{5}(x, y, \xi, d \xi)\right]
\end{aligned}
$$

and

$$
\begin{aligned}
d \sigma_{x x} & =2 G D_{x}(\xi)\left[2 T_{4}(x, y, \xi, d \xi)+y T_{6}(x, y, \xi, d \xi)\right] \\
& +2 G D_{y}(\xi)\left[-T_{5}(x, y, \xi, d \xi)+y T_{7}(x, y, \xi, d \xi)\right] \\
d \sigma_{y y} & =2 G D_{x}(\xi)\left[-y T_{6}(x, y, \xi, d \xi)\right] \\
& +2 G D_{y}(\xi)\left[-T_{5}(x, y, \xi, d \xi)-y T_{7}(x, y, \xi, d \xi)\right] \\
d \sigma_{x y} & =2 G D_{x}(\xi)\left[-T_{5}(x, y, \xi, d \xi)+y T_{7}(x, y, \xi, d \xi)\right] \\
& +2 G D_{y}(\xi)\left[-y T_{6}(x, y, \xi, d \xi)\right]
\end{aligned}
$$

$\mathrm{G}$ and $\mathrm{v}$ in these equations are shear modulus and Poisson's ratio, respectively. Functions F2 through F7 are described in Ref. [15]. Eqs (3) and (4) are used by Crouch and Starfield[14] to set up a constant displacement discontinuity boundary element method.

The solution to the subject problem is given by Crouch and Starfield [14]. The displacements and stresses can be written as

$$
\begin{aligned}
& u_{x}=D_{x}\left[2(1-v) F_{3}(x, y)-y F_{5}(x, y)\right]+D_{y}\left[-(1-2 v) F_{2}(x, y)-y F_{4}(x, y)\right], \\
& u_{y}=D_{x}\left[(1-2 v) F_{2}(x, y)-y F_{4}(x, y)\right]+D_{y}\left[2(1-v) F_{3}(x, y)-y F_{5}(x, y)\right],
\end{aligned}
$$

and

$$
\begin{aligned}
& \sigma_{x x}=2 G D_{x}\left[2 F_{4}(x, y)+y F_{6}(x, y)\right]+2 G D_{y}\left[-F_{5}(x, y)+y F_{7}(x, y)\right], \\
& \sigma_{y y}=2 G D_{x}\left[-y F_{6}(x, y)\right]+2 G D_{y}\left[-F_{5}(x, y)-y F_{7}(x, y)\right], \\
& \sigma_{x y}=2 G D_{x}\left[-F_{5}(x, y)+y F_{7}(x, y)\right]+2 G D_{y}\left[-y F_{6}(x, y)\right] .
\end{aligned}
$$

Function $f(x, y)$ in these equations can be written as :

$$
f(x, y)=\frac{-1}{4 \pi(1-v)}\left[y\left(\arctan \frac{y}{x-a}-\arctan \frac{y}{x+a}\right)-(x-a) \ln \sqrt{\left[(x-a)^{2}+y^{2}\right]}+(x+a) \ln \sqrt{\left[(x-a)^{2}+y^{2}\right]}\right]
$$

\section{Crack-Tip Displacement Discontinuity Elements}

By using the Eqs (3) and (4), recently, Yan [13] presented crack-tip displacement discontinuity elements, which can be classified as the left and the right crack-tip displacement discontinuity elements to deal with crack problems in general plane elasticity. The following gives basic formulas of the left upaccording to the manner in which discontinuity elements is crack-tip displacement discontinuity element.For the left crack-tip displacement discontinuity element (see Fig.2), its displacement discontinuity functions are chosen as

$$
D_{x}=H_{s}\left(\frac{a_{t i p}+\xi}{a_{t i p}}\right)^{\frac{1}{2}}, \quad D_{y}=H_{n}\left(\frac{a_{t i p}+\xi}{a_{t i p}}\right)^{\frac{1}{2}} .
$$




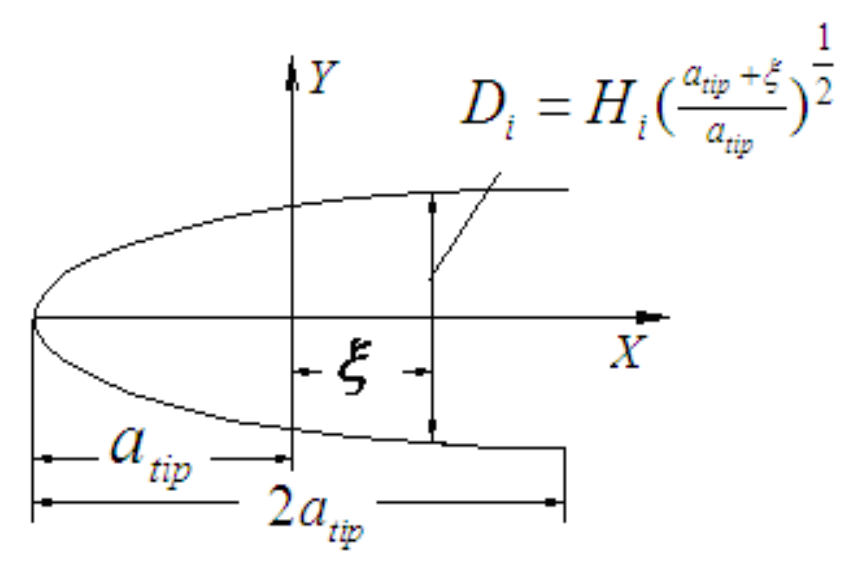

Fig.2 Schematic of the left crack tip displacement discontinuity element

where $\mathrm{Hs}$ and $\mathrm{Hn}$ are the tangential and normal displacement discontinuity quantities at the center of the element, respectively, atip is a half length of crack-tip element. Here, it is noted that the element has the same unknowns as the two-dimensional constant displacement discontinuity element. But it can be seen that the displacement discontinuity functions defined in (7) can model the displacement fields around the crack tip. The stress field determined by the displacement discontinuity functions (8) possesses $r-1 / 2$ singularity around the crack tip.

Based on the Eqs (3) and (4), the displacements and stresses at a point (x,y) due to the left crack-tip displacement discontinuity element can be obtained,

$$
\begin{aligned}
& u_{x}=H_{s}\left[2(1-v) B_{3}(x, y)-y B_{5}(x, y)\right]+H_{n}\left[-(1-2 v) B_{2}(x, y)-y B_{4}(x, y)\right], \\
& u_{y}=H_{s}\left[(1-2 v) B_{2}(x, y)-y B_{4}(x, y)\right]+H_{n}\left[2(1-v) B_{3}(x, y)-y B_{5}(x, y)\right], \\
& \text { and } \\
& \sigma_{x x}=2 G H_{s}\left[2 B_{4}(x, y)+y B_{6}(x, y)\right]+2 G H_{n}\left[-B_{5}(x, y)+y B_{7}(x, y)\right], \\
& \sigma_{y y}=2 G H_{s}\left[-y B_{6}(x, y)\right]+2 G H_{n}\left[-B_{5}(x, y)-y B_{7}(x, y)\right], \\
& \sigma_{x y}=2 G H_{s}\left[-B_{5}(x, y)+y B_{7}(x, y)\right]+2 G H_{n}\left[-y B_{6}(x, y)\right],
\end{aligned}
$$

where functions $B_{2}$ through $B_{7}$ are described in Ref. [16].

\section{Computational Formulas of the Stress Intensity Factors}

The objective of many analyses of linear elastic crack problems is to obtain the SIFs KI and KII. Based on the displacement field around the crack tip, the following formulas exist

$$
K_{\mathrm{I}}=-\frac{\sqrt{2 \pi} G H_{n}}{4(1-v) \sqrt{a_{t i p}}}, \quad K_{\mathrm{II}}=-\frac{\sqrt{2 \pi} G H_{s}}{4(1-v) \sqrt{a_{t i p}}} .
$$

\section{Two Parallel Surface Cracks with the Same Length 2a and the Same Depth h}

Shown in Fig. 3 is a planform of two parallel surface cracks with the same length $2 \mathrm{a}$ and the same depth $\mathrm{h}$ subjected to uniform stress $\sigma$ at infinity. For this crack problem, the symmetry condition can be used. The following geometry parameters are considered

$$
a / d=0.05,0.1,0.2,0.3,0.4,0.5,0.6,0.7,0.8,0.9 \quad h / a=1.0,1.1,1.2,1.3,1.4,1.6,1.8,2.0
$$

Regarding discretization, 20 boundary elements with a same size, $a / 20$, are discretized for a half of the crack $A B$ shown in Fig.3. The calculated SIFs normalized by $\sigma \sqrt{\pi a}$ are given in Fig.4.

(a) When the distance between the two surface cracks is very large, for example, $2 a / d=0.02$, the normalized SIFs at the crack tip $B$ are not affected by the surface crack $A$ ' $B$ ' no matter what $h / a$ is large or small. At this time, the normalized SIFs at the crack tip $B$ calculated by the present numerical approach ought to agree with those predicted by using (9). 
(b) With increase of $h / a$, the surface cracks $A B$ and $A{ }^{\prime} B$ ' tend to penetrated cracks. When $h / a$ reaches 2.0, the surface cracks $A B$ and $A^{\prime} B^{\prime}$ ' can be regarded as penetrated cracks. At this time, the normalized SIFs at the crack tip $B$ calculated by the present numerical approach ought to agree with those of the two parallel penetrated cracks.

The two points above illustrate the accuracy of the present numerical approach for analyzing such a surface crack problem shown in Fig.4.

The calculated normalized SIFs at the crack tip $B$ given listed in Fig.4. can be used to reveal the effect of geometry parameters, $2 a / d$ and $h / a$, on the normalized SIFs. In order to conveniently observe this effect, Fig. 4 shows variation of the normalized SIFs at the crack tip $B$ with $h / a$ for three cases: $2 a / d=0.02,1.0$ and 5.0 .

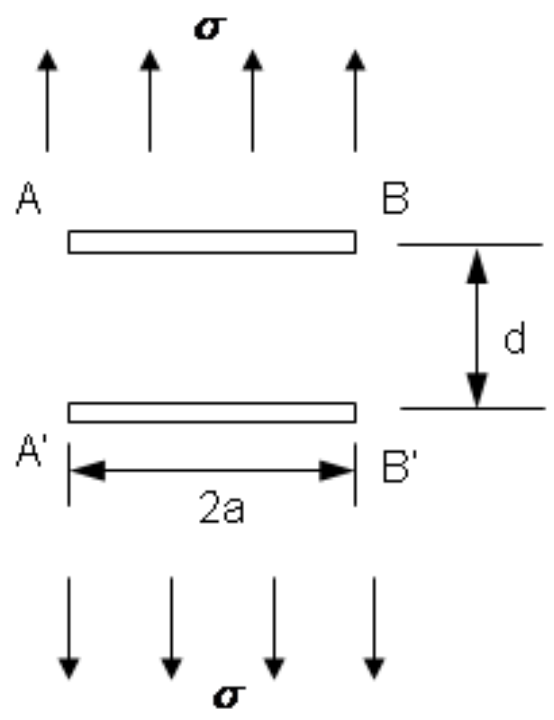

Fig.3 Schematic of a planform of two parallel surface cracks

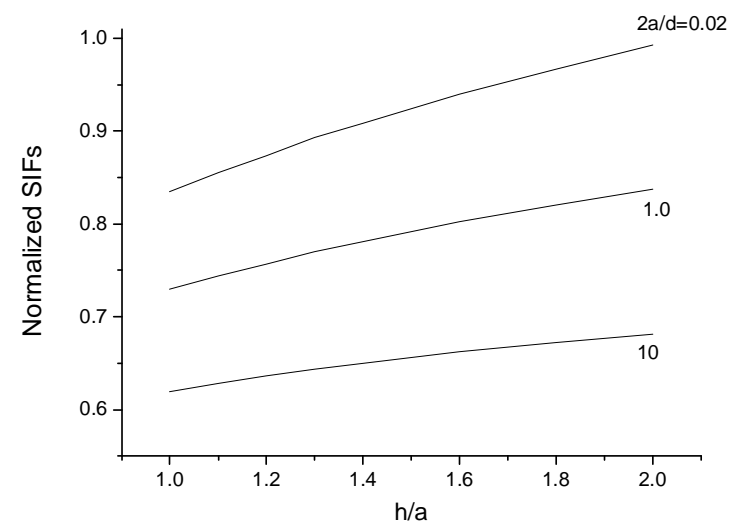

Fig.4 Variation of normalized SIFs at the crack tip $B$ with $h / a$

\section{Conclusions}

A numerical approach for two Parallel Surface Cracks of infinite plane was presented in this paper. Numerical examples showed that the numerical approach is simple, yet accurate for calculating the SIFs of two Parallel Surface Cracks of infinite plane. When the distance between the two surface cracks is very large, the normalized SIFs at the crack tips $A$ and $B$ are not affected by the surface crack $A^{\prime} B^{\prime}$ and $F_{\mathrm{A}} \approx F_{\mathrm{B}}$ no matter what $h / a$ is large or small. With increase of $h / a$, the surface cracks $A B$ and $A^{\prime} B$ ' tend to penetrated cracks. 


\section{Acknowledgement}

Special thanks are due to the National Natural Science Foundation of China (No. 11572113), the talent project of Harbin City (2015RAQXJ005) and Young talent project of Heilongjiang University of Science and Technology for supporting the present work.

\section{References}

1. Murakami, Y., Stress Intensity Factors Handbook, Pergamon Press, New York, 1987.

2. Liu, B. Yan, L. and Yan, X., An Elliptical Hole-Crack Problem in Infinite Plate Subjected to Internal Pressure, Journal of Liaoning Technical University.2012, 31 (4) :540-543.

3. Yan, X., Interaction of arbitrary multiple cracks in an infinite plate, $J$ Strain Analysis for Engineering Design, 39 (3), 237-244(2004).

4. Pan, E., A General Boundary Element Analysis of 2-D Linear Elastic Fracture Mechanics, Int J Fract 88,41-59(1997).

5. Liu, B. Yan, X., Triangle hole-crack problem in infinite plate in tension, J. of harbin institute technology. 2011,43(7):51-56.

6. Yan, X., A numerical analysis of cracks emanating from an elliptical hole in a 2-D elasticity plate, European J of Mechanics-A, 25 (1), 142-153(2006).

7. Bowie, O. L., Analysis of an infinite plate containing radial cracks originating at the boundary of an internal circular hole, J. Math. Phys, 1956, 35, 60-71.

8. Newman, J.C. Jr., An improved method of collocation for the stress analysis of cracked plates with various shaped boundaries, NASA TN, D-6376, 1971,1-45.

9. Newman,J.C.Jr, Bigelow, C.A. and Shivakumar, K.N. Three-dimensional elastic-plastic finite element analyses of constraint variations in cracked bodies, Eng. Fract. Mech., 46(1), 1-13 (1993).

10. Shivakumar K.N. and Newman, J.C.Jr, Stress intensity factors for large aspect ratio surface and corner cracks at a semi-circular notch in a tension specimen, Eng. Fract. Mech. 38(6), 467-473(1991).

11. Crouch, S. L. and Starfield, A. M., Boundary Element Method in Solid Mechanics, with Application in Rock Mechanics and Geological Mechanics, London, Geore Allon \& Unwin, Bonton, Sydney, 1983,1-220.

12. Scavia,C., A numerical technique for the analysis of cracks subjected to normal compressive stresses, Int J Num Methods Eng 33, 929-942(1992).

13. Yan, X., An effective numerical approach for multiple void-crack interaction, ASME J. Appl. Mech. 73 (4), 525-535(2006).

14. Crouch, S. L. and Starfied, A. M., Boundary Element Method in Solid Mechanics, with Application in Rock Mechanics and Geological Mechanics, London, Geore Allon \& Unwin, Bonton, Sydney, 1983.

15. Yan, X., Liu B. and Hu Z., Fatigue paths simulation of a center slant crack in cracked brazilian disk by BEM, Mechanica. 2010,42(6):1331-1236. 